\title{
ON THE HOCHSCHILD COHOMOLOGY OF TAME HECKE ALGEBRAS
}

\author{
KARIN ERDMANN AND SIBYLLE SCHROLL
}

\begin{abstract}
We explicitly calculate a projective bimodule resolution for a special biserial algebra giving rise to the Hecke algebra $\mathcal{H}_{q}\left(S_{4}\right)$ when $q=-1$. We then determine the dimensions of the Hochschild cohomology groups.
\end{abstract}

\section{INTRODUCTION}

In this paper we are interested in Hochschild cohomology of finite-dimensional algebras; the main motivation is to generalize group cohomology to larger classes of algebras. If suitable finite generation holds, one can define support varieties of modules as introduced by [SS]. Furthermore, when the algebra is self-injective, many of the properties of group representations generalize to this setting as was shown in [EHSST]. Although Hecke algebras do not have a Hopf algebra structure, one may expect that being deformations of group algebras, they should have good homological properties. Furthermore, the study of Hochschild cohomology for blocks of group algebras with cyclic or dihedral defect groups has made use of the fact that the basic algebras in this case are special biserial [Ho1, Ho2]. These results suggest that Hochschild cohomology of special biserial algebras might be accessible more generally.

This paper is concerned with the self-injective special biserial algebra $A$ which occurs as the basic algebra of the Hecke algebra $\mathcal{H}_{q}\left(S_{4}\right)$ when $q=-1$. For this algebra we will explicitly give a minimal projective bimodule resolution and use it to calculate the dimensions of the Hochschild cohomology. By [EN] any tame block of some Hecke algebra $\mathcal{H}_{q}\left(S_{n}\right)$ is derived equivalent to $A$. Since Hochschild cohomology is invariant under derived equivalence, our result gives information for arbitrary tame blocks Hecke algebras of type $A$.

In order for the Hochschild cohomology ring to be finitely generated, the dimensions of $H H^{n}(A)$ must have at most polynomial growth. Therefore our results give evidence towards the finite generation of the Hochschild cohomology. Further evidence towards this comes from [ES], which shows by a different approach, via derived equivalence, that the finite generation hypothesis in [EHSST] holds for the special biserial algebra under consideration in this paper.

The second author acknowledges support through an EPSRC Postdoctoral Fellowship EP/D077656/1 as well as through a Leverhulme Early Career Fellowship.

2000 Mathematics Subject Classification: 16E40, 20C08, 16E10 (primary). 
The algebra $A$ can be described by a quiver and relations (cf. [EN]). Let $k$ be a field and let $\mathcal{Q}$ be the quiver described in figure 1 with relations

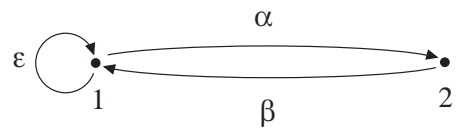

Figure 1 . The quiver of $A$

$$
\varepsilon \alpha=\beta \varepsilon=0,(\alpha \beta)^{2}=\varepsilon^{2} .
$$

Then $A=k \mathcal{Q} / I$ where $I$ is generated by the relations in (1). We denote the trivial path at the vertex $i, i=1,2$, by $e_{i}$ and the corresponding simple $A$-module by $S_{i}$. We read paths from left to right and, unless stated differently, all the modules considered will be (finitely generated) right $A$-modules. Let $\operatorname{rad} A$ be the Jacobson radical of $A$ and denote the enveloping algebra of $A$ by $A^{e}=A^{o p} \otimes_{k} A$. For a path $a$ in $A$ we denote by $\mathbf{o}(a)$ the idempotent at the vertex from which the path leaves and by $\mathbf{t}(a)$ the idempotent at the vertex at which the path arrives. We say a path $p$ in $k \mathcal{Q}$ is right (resp. left) uniform if it is a linear combination of paths ending in (resp. beginning at) a single vertex.

\section{Construction of a minimal projective Bimodule Resolution}

In this section we construct a minimal projective $A$ - $A$-bimodule resolution of $A$, that is, we construct an exact complex

$$
R_{\bullet}: \quad \ldots \longrightarrow R_{n} \stackrel{\delta_{n}}{\longrightarrow} R_{n-1} \stackrel{\delta_{n-1}}{\longrightarrow} \ldots \longrightarrow R_{1} \stackrel{\delta_{1}}{\longrightarrow} R_{0} \stackrel{\delta_{0}}{\longrightarrow} A \longrightarrow 0
$$

with $R_{n}=\bigoplus P_{i j}$ and where $P_{i j}$ is the projective $A$ - $A$-bimodule $A e_{i} \otimes e_{j} A$. By [H] the projective $P_{i j}$ occurs in $R_{n}$ exactly as many times as $\operatorname{dimExt}^{n}\left(S_{i}, S_{j}\right)$.

Minimal projective resolutions of simple modules in this case can be calculated, for example similar to the Appendix in $[\mathrm{R}]$. Thus the terms of the projective bimodule resolution $\left(R_{\bullet}, \delta\right)$ are periodic of period 4 and, for $k \geq 0$,

$$
\begin{aligned}
& R_{4 k} \cong P_{11}^{2 k+1} \oplus P_{22} \\
& R_{4 k+1} \cong P_{11}^{2 k+1} \oplus P_{12} \oplus P_{21} \\
& R_{4 k+2} \cong P_{11}^{2 k+1} \oplus P_{12} \oplus P_{21} \\
& R_{4 k+3} \cong P_{11}^{2 k+2} \oplus P_{22} .
\end{aligned}
$$


2.1. From one-sided to two-sided resolutions. The construction of the differential $\delta$ is based on the construction of a minimal projective resolution of $A / \operatorname{rad} A$ as a left and as a right $A$-module as developed in [GSZ]. We then use the differentials of the left and right module resolutions of $A / \operatorname{rad} A$ to explicitly construct the differential of a minimal $A$ - $A$-bimodule resolution of $A$. Our approach follows that of [GS] (see also [ST] ) and, for Koszul algebras, that of [GHMS.

We begin by briefly recalling the construction of projective resolutions as developed in [GSZ]. Let $\Lambda$ be a finite dimensional algebra of the form $\Lambda=k Q / I$. In [GSZ the authors then define sets of paths $g^{n}$ in $k Q$ for $n \geq 0$ where $g^{0}$ is given by the trivial paths in $k Q, g^{1}$ corresponds to the arrows of $Q$ and $g^{2}$ is a minimal set of uniform generators of $I$. For $n \geq 3, g^{n}$ is constructed recursively such that the elements in $g^{n}$ are uniform and satisfy the property: for $x \in g^{n}, x=\sum_{p \in g^{n-1}} p \lambda_{p}=\sum_{q \in g^{n-2}} q \mu_{q}$ for unique elements $\lambda_{p}, \mu_{q} \in k Q$ and $\mu_{q} \in I$ such that there is a minimal projective $\Lambda$-module resolution

$P_{\bullet}: \quad \ldots \longrightarrow P_{n} \stackrel{d_{n}}{\longrightarrow} P_{n-1} \stackrel{d_{n-1}}{\longrightarrow} \ldots \longrightarrow P_{1} \stackrel{d_{1}}{\longrightarrow} P_{0} \longrightarrow \Lambda / \operatorname{rad} \Lambda \longrightarrow 0$

such that

(1) $P_{n}=\bigoplus_{x \in g^{n}} \mathbf{t}(x) \Lambda$ for all $n \geq 0$

(2) the $\operatorname{map}_{n}: P_{n} \rightarrow P_{n-1}$ is given by

$$
\mathbf{t}(x) a \mapsto \sum_{p \in g^{n-1}} \lambda_{p} \mathbf{t}(x) a
$$

where as above $x=\sum_{p \in g^{n-1}} p \lambda_{p}$ is determined by unique elements $\lambda_{p} \in k Q$.

The collection of elements of $g^{n}$ is written as $g^{n}=\left\{g_{i}^{n}\right\}$.

In [GS] the sets $g^{n}$ are then used to give an explicit construction of the first three differentials of a minimal projective $\Lambda$ - $\Lambda$-bimodule resolution of $\Lambda$. Based on this approach we first construct the sets $g^{n}$ for the minimal right $A$-module resolution of $A / \mathrm{radA}$ and then proceed to construct a $A$ - $A$-bimodule resolution of $A$.

2.2. A minimal projective right $A$-module resolution of $A / \operatorname{rad} A$. In this paragraph we construct a minimal projective right $A$-module resolution of $A / \operatorname{rad} A$. To facilitate the notations we slightly change the notation of [GSZ] and define the sets defining the differentials as follows:

Definition 2.1. For the algebra $A$ we set $g_{1}^{0}=e_{1}$ and $f_{22}^{0}=e_{2}$ and we define recursively, for all $i=0,1,2,3$ and all $k \geq 0$ and for all $n \geq 4$, 


$$
\begin{aligned}
& g_{r}^{n}= \begin{cases}g_{1}^{n-1} \varepsilon-g_{2}^{n-1} \alpha \beta & \text { if } r=1 \\
g_{r-1}^{n-1} \varepsilon-g_{r+1}^{n-1} \alpha \beta & \text { if } r \text { odd, } 1<r \leq 2 k-1 \\
g_{r+1}^{n-1} \varepsilon+g_{r-1}^{n-1} \alpha \beta & \text { if } r \text { even, } 1<r \leq 2 k\end{cases} \\
& g_{2 k+1}^{4 k+i}= \begin{cases}g_{2 k+1}^{4 k+i-1} \varepsilon & \text { if } i=1 \text { and } k=0 \\
g_{2 k+1}^{4 k+1} \varepsilon & \text { if } i=0,1 \text { and } k>0 \\
g_{2 k+1-1}^{4 k+1} \varepsilon-f_{12}^{4 k+i-1} \beta \alpha \beta & \text { if } i=2,3 \text { and } k=0 \\
g_{2 k}^{4 k+i-1} \varepsilon-f_{12}^{4 k+i-1} \beta \alpha \beta & \text { if } i=2,3 \text { and } k>0\end{cases} \\
& g_{2 k}^{4 k}=g_{2 k-1}^{4 k-1} \alpha \beta \quad \text { if } k>0 \\
& g_{2 k+2}^{4 k+3}=g_{2 k+1}^{4 k+2} \alpha \beta \quad \text { if } k>0 \\
& f_{12}^{4 k+i}=g_{2 k+1}^{4 k+i-1} \alpha \quad \text { if } i=1,2 \\
& f_{21}^{4 k+i}= \begin{cases}f_{22}^{4 k} \beta & \text { if } i=1 \\
f_{21}^{4 k+1} \varepsilon & \text { if } i=2\end{cases} \\
& f_{22}^{4 k+i}= \begin{cases}f_{22}^{4 k-1}(\beta \alpha)^{2} & \text { if } i=0 \\
f_{21}^{4 k+2} \alpha & \text { if } i=3\end{cases}
\end{aligned}
$$

$$
\text { . }
$$


resolution of $A$. But first we need to introduce some notation: we write the elements $e_{i} \otimes e_{j}$ of $A e_{i} \otimes e_{j} A$ as follows

$$
\begin{aligned}
& \mathfrak{a}:=e_{1} \otimes e_{1} \in P_{11}=A e_{1} \otimes e_{1} A \\
& \mathfrak{b}:=e_{1} \otimes e_{2} \in P_{12}=A e_{1} \otimes e_{2} A \\
& \mathfrak{c}:=e_{2} \otimes e_{1} \in P_{21}=A e_{2} \otimes e_{1} A \\
& \mathfrak{d}:=e_{2} \otimes e_{2} \in P_{22}=A e_{2} \otimes e_{2} A \text {. }
\end{aligned}
$$

The terms of $R_{\bullet}$ in each degree contain multiple copies of $P_{11}$ and always at most one copy of $P_{12}, P_{21}$ and $P_{22}$ (see (2) ). We need to distinguish the different copies of $P_{11}$ in $R_{n}$, for $n \geq 0$. We do this by adding superscripts. As usual, the subscripts determine in which degree of the complex an element lies. For example, $\mathfrak{a}_{i}^{r}=e_{1} \otimes e_{1}$ lies in the $r$ th copy of $P_{11}$ as a summand of $R_{i}, \mathfrak{a}_{i+1}^{r+1}=e_{1} \otimes e_{1}$ lies in the $r+1$ th copy of $P_{11}$ as a summand of $R_{i+1}$ and $\mathfrak{b}_{4 k+1}=e_{1} \otimes e_{2}$ (resp. $\mathfrak{c}_{4 k+1}=e_{2} \otimes e_{1}$ ) lies in the copy of $P_{12}$ (resp. $P_{21}$ ) as a summand of $R_{4 k+1}$.

Definition 2.3. The map $\delta_{0}: R_{0} \rightarrow A$ is given by multiplication. With the above notation, for $n \geq 1$, we define the differential $\delta_{n}: R_{n} \rightarrow R_{n-1}$ by the following recursively defined maps

$$
\mathfrak{a}_{n}^{r} \mapsto\left\{\begin{array}{lr}
\mathfrak{a}_{n-1}^{1} \varepsilon-\varepsilon \mathfrak{a}_{n-1}^{1} & n=1, r=1 \\
\mathfrak{a}_{n-1}^{1} \varepsilon-\mathfrak{b}_{n-1} \beta \alpha \beta+\varepsilon \mathfrak{a}_{n-1}^{1}-\alpha \beta \alpha \mathfrak{c}_{n-1}-\alpha \beta \mathfrak{b}_{n-1} \beta-\alpha \mathfrak{c}_{n-1} \alpha \beta & n=2, r=1 \\
\mathfrak{a}_{n-1}^{1} \varepsilon-\mathfrak{b}_{n-1} \beta \alpha \beta-\varepsilon \mathfrak{a}_{n-1}^{1}+\alpha \beta \alpha \mathfrak{c}_{n-1} & n=3, r=1 \\
\mathfrak{a}_{n-1}^{1} \alpha \beta-\alpha \beta \mathfrak{a}_{n-1}^{1}-\varepsilon \mathfrak{b}_{n-1} \beta+\alpha \mathfrak{c}_{n-1} \varepsilon & n=3, r=2
\end{array}\right.
$$

and, for all $n=4 k+i$ with $i=0,1,2,3$ and $k \geq 1$,

$$
\mathfrak{a}_{4 k+i}^{r} \mapsto \begin{cases}\mathfrak{a}^{1} \epsilon-\mathfrak{a}^{2} \alpha \beta+\epsilon \mathfrak{a}^{1}-\alpha \beta \mathfrak{a}^{2} & \text { if } r=1 \text { and } i=0,2 \\ \mathfrak{a}^{r+1} \varepsilon+\mathfrak{a}^{r-1} \alpha \beta+\varepsilon \mathfrak{a}^{r}-\alpha \beta \mathfrak{a}^{r+2} & \text { if } 1<r<2 k, r \text { even and } i=0,2 \\ \mathfrak{a}^{r-1} \varepsilon-\mathfrak{a}^{r+1} \alpha \beta+\varepsilon \mathfrak{a}^{r}+\alpha \beta \mathfrak{a}^{r-2} & \text { if } 1<r<2 k, r \text { odd and } i=0,2 \\ \mathfrak{a}^{r-1} \alpha \beta+\varepsilon \mathfrak{a}^{r}+(-1)^{k} \alpha \beta \alpha \mathfrak{d} \beta & \text { if } r=2 k \text { and } i=0 \\ \mathfrak{a}^{r-1} \varepsilon+\alpha \beta \mathfrak{a}^{r-2}+(-1)^{k} \alpha \mathfrak{d} \beta \alpha \beta & \text { if } r=2 k+1 \text { and } i=0 \\ \mathfrak{a}^{r+1} \varepsilon+\mathfrak{a}^{r-1} \alpha \beta+\varepsilon \mathfrak{a}^{r}+(-1)^{k+1} \alpha \beta \alpha \mathfrak{c}-\alpha \beta \mathfrak{b} \beta & \text { if } r=2 k \text { and } i=2 \\ \mathfrak{a}^{r-1} \varepsilon+\varepsilon \mathfrak{a}^{r}+\alpha \beta \mathfrak{a}^{r-2}-\mathfrak{b} \beta \alpha \beta+(-1)^{k+1} \alpha \mathfrak{c} \alpha \beta & \text { if } r=2 k+1 \text { and } i=2 \\ & \text { if } r=1 \text { and } i=1,3 \\ \mathfrak{a}^{1} \varepsilon-\mathfrak{a}^{2} \alpha \beta-\varepsilon \mathfrak{a}^{1}+\alpha \beta \mathfrak{a}^{3} & \text { if } r=2 \text { and } i=1,3 \\ \mathfrak{a}^{3} \varepsilon+\mathfrak{a}^{1} \alpha \beta-\varepsilon \mathfrak{a}^{2}-\alpha \beta \mathfrak{a}^{1} & \text { if } 1<r \leq 2 k \text { and } r \text { is odd } \\ \mathfrak{a}^{r-1} \varepsilon-\mathfrak{a}^{r+1} \alpha \beta-\varepsilon \mathfrak{a}^{r}+\alpha \beta \mathfrak{a}^{r+2} & \text { if } 1<r \leq 2 k \text { and } r \text { is even } \\ \mathfrak{a}^{r+1} \varepsilon+\mathfrak{a}^{r-1} \alpha \beta-\varepsilon \mathfrak{a}^{r}-\alpha \beta \mathfrak{a}^{r-2} & \text { if } r=2 k+1 \text { and } i=1 \\ \mathfrak{a}^{r-1} \varepsilon-\varepsilon \mathfrak{a}^{r} & \text { if } r=2 k+1 \text { and } i=3 \\ \mathfrak{a}^{r-1} \varepsilon-\mathfrak{b} \beta \alpha \beta-\varepsilon \mathfrak{a}^{r}+(-1)^{k} \alpha \beta \alpha \mathfrak{c} & \text { if } r=2 k+2 \text { and } i=3 \\ \mathfrak{a}^{r-1} \alpha \beta-\alpha \beta \mathfrak{a}^{r-2}+(-1)^{k} \alpha \mathfrak{c} \varepsilon-\varepsilon \mathfrak{b} \beta & \end{cases}
$$

where on the right hand side we omit the subscript with the understanding that these are all elements in $R_{4 k+i-1}$. Finally, for $n=4 k+i$ with $i=0,1,2,3$ and $k \geq 0$, we 
set

$$
\begin{aligned}
\mathfrak{d}_{4 k} & \mapsto \mathfrak{d}_{4 k-1}(\beta \alpha)^{2}+(\beta \alpha)^{2} \mathfrak{d}_{4 k-1}+(-1)^{k} \beta \mathfrak{a}_{4 k-1}^{2 k-1} \alpha \\
\mathfrak{b}_{4 k+1} & \mapsto \mathfrak{a}_{4 k}^{2 k+1} \alpha+(-1)^{k+1} \alpha \mathfrak{d}_{4 k} \\
\mathfrak{c}_{4 k+1} & \mapsto \mathfrak{d}_{4 k} \beta+(-1)^{k+1} \beta \mathfrak{a}_{4 k}^{2 k} \\
\mathfrak{b}_{4 k+2} & \mapsto \mathfrak{a}_{4 k+1}^{2 k+1} \alpha+\varepsilon \mathfrak{b}_{4 k+1} \\
\mathfrak{c}_{4 k+2} & \mapsto \mathfrak{c}_{4 k+1} \varepsilon+(-1)^{k} \beta \mathfrak{a}_{4 k+1}^{2 k+1} \\
\mathfrak{d}_{4 k+3} & \mapsto \mathfrak{c}_{4 k+2} \alpha+(-1)^{k+1} \beta \mathfrak{b}_{4 k+2} .
\end{aligned}
$$

It is easily checked that if we set $R_{n}=\bigoplus_{x \in g^{n}} A \mathbf{o}(x) \otimes \mathbf{t}(x) A$ we obtain exactly the expressions as stated at the beginning of section 2. Furthermore, we have that,

Theorem 2.4. The complex $\left(R_{\bullet}, \delta\right)$ is a minimal projective resolution of $A$ as an A-A-bimodule.

Proof: It is straightforward to verify that $\operatorname{Im} \delta_{n} \subseteq \operatorname{Ker} \delta_{n-1}$ and therefore $\left(R_{\bullet}, \delta\right)$ is a complex.

In order to show that the complex is exact we first note that there is an isomorphism of complexes of right $A$-modules $\left(A / \operatorname{rad} A \otimes_{A} R_{\bullet}, i d \otimes \delta\right) \simeq\left(P_{\bullet}, d\right)$. This holds since it is easily verified that for all $n$, we have $A / \operatorname{rad} A \otimes_{A} R_{n} \simeq P_{n}$ and furthermore, the diagram

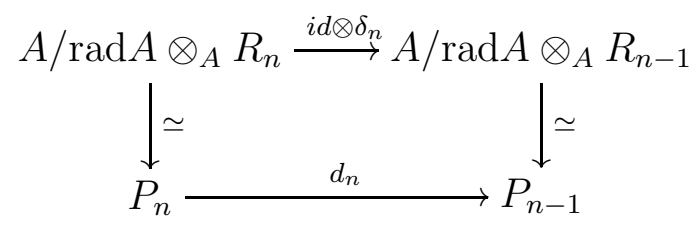

commutes for all $n \geq 1$. Now we apply the same arguments as in [GS, Proposition 2.8] and see also [ST, Theorem 1.6]. Suppose that $\operatorname{Ker} \delta_{n-1} \nsubseteq \mathbb{I m} \delta_{n}$ for some $n \geq 1$. Then there exists a simple $A$ - $A$-bimodule $S \otimes T$ composed of a simple left $A$-module $S$ and a simple right $A$ module $T$ and there exists a non-zero map $f: \operatorname{Ker} \delta_{n-1} \rightarrow S \otimes T$. We have seen (Proposition 2.2 and (3) ) that the complex $\left(A / \operatorname{rad} A \otimes_{A} R_{\bullet}, i d \otimes \delta\right)$ is a minimal projective resolution of $A / \operatorname{rad} A$ as a right $A$-module. Therefore the following isomorphisms of right $A$-modules hold $A / \operatorname{rad} A \otimes_{A} \operatorname{Im} \delta_{n} \simeq \operatorname{Im}\left(i d \otimes \delta_{n}\right) \simeq$ $\operatorname{Ker}\left(i d \otimes \delta_{n-1}\right) \simeq A / \operatorname{rad} A \otimes_{A} \operatorname{Ker} \delta_{n-1}$ and we obtain a non-zero map as the composition

$A / \mathrm{rad} A \otimes_{A} R_{n} \stackrel{i d \otimes \delta_{n}}{\longrightarrow} A / \mathrm{rad} A \otimes_{A} \operatorname{Im} \delta_{n} \stackrel{\simeq}{\longrightarrow} A / \operatorname{rad} A \otimes_{A} \operatorname{Ker} \delta_{n-1} \stackrel{i d \otimes f}{\longrightarrow} S \otimes T$. 
But this is the same as the functor $A / \operatorname{rad} A \otimes_{A}$ - applied to the sequence of maps

$R_{n} \stackrel{\delta_{n}}{\rightarrow} \operatorname{Ker} \delta_{n-1} \stackrel{f}{\rightarrow} S \otimes T$. However, the map $f \circ \delta_{n}$ is zero. This gives a contradiction and therefore the complex $\left(R_{\bullet}, \delta_{n}\right)$ is exact.

Since we calculated the terms of $R_{\bullet}$ following $[\mathrm{H}]$, the minimality follows.

\section{Dimensions of the Hochschild Cohomology groups}

The Hochschild cohomology of $A$ is defined by $\operatorname{HH}^{*}(A)=\operatorname{Ext}_{A^{e}}^{*}(A, A)$. In each degree $n, n \geq 0$, there is an exact sequence

$0 \rightarrow \operatorname{Hom}_{A^{e}}\left(\Omega^{n}(A), A\right) \rightarrow \operatorname{Hom}_{A^{e}}\left(R_{n}, A\right) \rightarrow \operatorname{Hom}_{A^{e}}\left(\Omega^{n+1}(A), A\right) \rightarrow \operatorname{Ext}_{A^{e}}^{n+1}(A, A) \rightarrow 0$.

and since we have an explicit description of the terms $R_{n}$ of the $A$-A-bimodule resolution of $A$, it is straightforward to calculate the dimensions of the complex $\operatorname{Hom}_{A^{e}}\left(R^{\bullet}, A\right)$. These dimensions are for, $k \geq 0$,

$$
\begin{aligned}
& \operatorname{dim} \operatorname{Hom}_{A^{e}}\left(R_{4 k}, A\right) \quad=8 k+7 \\
& \operatorname{dim} \operatorname{Hom}_{A^{e}}\left(R_{4 k+1}, A\right)=8 k+8 \\
& \operatorname{dim} \operatorname{Hom}_{A^{e}}\left(R_{4 k+2}, A\right)=8 k+8 \\
& \operatorname{dim} \operatorname{Hom}_{A^{e}}\left(R_{4 k+3}, A\right)=8 k+11 \text {. }
\end{aligned}
$$

Furthermore, $\operatorname{HH}^{0}(A)$ is given by the center $Z(A)$ of $A$. As an algebra, $Z(A)$ is generated by

$$
\left\{e_{1}+e_{2}, \varepsilon, \alpha \beta+\beta \alpha, \varepsilon^{2}, \beta \alpha \beta \alpha\right\} .
$$

By (44), in order to calculate the dimensions of $\operatorname{HH}^{n}(A)$ for all $n \geq 0$ we need to calculate the dimensions of $\operatorname{Hom}_{A^{e}}\left(\Omega^{n}(A), A\right)$ for all $n \geq 1$. Using the description

$$
\operatorname{Hom}_{A^{e}}\left(\Omega^{n}(A), A\right)=\left\{\gamma_{n} \in \operatorname{Hom}_{A^{e}}\left(R_{n}, A\right) \mid \gamma_{n}\left(\Omega(A)^{n+1}\right)=0\right\}
$$

we will calculate a basis for $\operatorname{Hom}_{A^{e}}\left(\Omega^{n}(A), A\right)$. Since $R_{\bullet}$ is exact the images of the differential yield the generators of $\Omega^{n}(A)$ in each degree $n$. Thus, for $k \geq 0$, we have

$$
\begin{aligned}
& \Omega^{4 k}(A)=<\delta_{4 k}\left(\mathfrak{a}_{4 k}^{1}\right), \ldots, \delta_{4 k}\left(\mathfrak{a}_{4 k}^{2 k+1}\right), \delta_{4 k}\left(\mathfrak{d}_{4 k}\right)> \\
& \Omega^{4 k+1}(A)=<\delta_{4 k+1}\left(\mathfrak{a}_{4 k+1}^{1}\right), \ldots, \delta_{4 k+1}\left(\mathfrak{a}_{4 k+1}^{2 k+1}\right), \delta_{4 k+1}\left(\mathfrak{b}_{4 k+1}\right), \delta_{4 k+1}\left(\mathfrak{c}_{4 k+1}\right)> \\
& \Omega^{4 k+2}(A)=<\delta_{4 k+2}\left(\mathfrak{a}_{4 k+2}^{1}\right), \ldots, \delta_{4 k+2}\left(\mathfrak{a}_{4 k+2}^{2 k+1}\right), \delta_{4 k+2}\left(\mathfrak{b}_{4 k+2}\right), \delta_{4 k+2}\left(\mathfrak{c}_{4 k+2}\right)> \\
& \Omega^{4 k+3}(A)=<\delta_{4 k+3}\left(\mathfrak{a}_{4 k+3}^{1}\right), \ldots, \delta_{4 k+3}\left(\mathfrak{a}_{4 k+3}^{2 k+2}\right), \delta_{4 k+3}\left(\mathfrak{d}_{4 k+3}\right)>.
\end{aligned}
$$

For each of the four cases above, we will obtain a system of equations whose solution space gives a basis of $\operatorname{Hom}_{A^{e}}\left(\Omega^{n}(A), A\right)$. 
3.1. Characteristic of $k \neq 2$. Suppose that the characteristic of $k \neq 2$. Then

Lemma 3.1. We have the following dimensions for $k \leq 0$ :

$$
\operatorname{dim} \operatorname{Hom}_{A^{e}}\left(\Omega^{4 k+i}(A), A\right)= \begin{cases}5 k+5 & \text { if } i=0 \\ 5 k+5 & \text { if } i=1 \\ 5 k+6 & \text { if } i=2 \\ 5 k+6 & \text { if } i=3\end{cases}
$$

Proof: Let $k \geq 1$, we start by calculating the dimension of $\operatorname{Hom}_{A^{e}}\left(\Omega^{4 k}(A), A\right)$. Let $\gamma_{4 k}: R_{4 k} \rightarrow A$ be the map defined by $\gamma_{4 k}\left(\mathfrak{a}_{4 k}^{j}\right)=a_{1}^{j} e_{1}+b_{1}^{j} \varepsilon+c_{1}^{j} \alpha \beta+d_{1}^{j} \varepsilon^{2}$ for $1 \geq$ $j \geq 2 k+1$ and $\gamma_{4 k}\left(\mathfrak{d}_{4 k}\right)=a_{2} e_{2}+b_{2} \beta \alpha+c_{2} \beta \alpha \beta \alpha$ with $a_{1}^{j}, b_{1}^{j}, \ldots, a_{2}, b_{2} \in k$. With this notation we have that $\gamma_{4 k}(x)=0$ for all generators $x$ of $\Omega^{4 k+1}(A)$ if and only if for all even $n$, such that $2 \leq n \leq 2 k$,

$$
a_{1}^{n}=a_{1}^{n+1} \quad b_{1}^{n}=b_{1}^{n+1} \quad c_{1}^{n}=c_{1}^{n+1}
$$

and

$$
(-1)^{k+1} a_{2}=a_{1}^{2 k+1} \quad(-1)^{k+1} b_{2}=c_{1}^{2 k+1} .
$$

Thus the dimension of $\operatorname{Hom}_{A^{e}}\left(\Omega^{4 k}(A), A\right)$ is $5 k+5$.

Similarly for the dimension of $\operatorname{Hom}_{A^{e}}\left(\Omega^{4 k+1}(A), A\right)$, let $\gamma_{4 k+1}: R_{4 k+1} \rightarrow A$ be the map defined by $\gamma_{4 k+1}\left(\mathfrak{a}_{4 k+1}^{i}\right)=a_{1}^{i} e_{1}+b_{1}^{i} \varepsilon+c_{1}^{i} \alpha \beta+d_{1}^{i} \varepsilon^{2}$ for $1 \geq i \geq 2 k+1$ and $\gamma_{4 k+1}\left(\mathfrak{b}_{4 k+1}\right)=$ $a_{12} \alpha+b_{12} \alpha \beta \alpha$ and $\gamma_{4 k+1}\left(\mathfrak{c}_{4 k+1}\right)=a_{21} \beta+b_{21} \beta \alpha \beta$ with $a_{1}^{i}, b_{1}^{i}, \ldots, a_{12}, b_{21}, \ldots \in k$. Then for all generators $x$ of $\Omega^{4 k+2}(A)$, we have that $\gamma_{4 k+1}(x)=0$ if and only if the following equations hold

$$
\begin{gathered}
a_{1}^{1}=a_{1}^{2}=\ldots=a_{1}^{2 k+1}=a_{1}^{2 k+1}=0 \\
b_{1}^{1}=c_{1}^{2} \\
b_{1}^{2 k}+b_{1}^{2 k+1}+c_{1}^{2 k-1}-a_{12}+(-1)^{k+1} a_{21}=0 \\
b_{1}^{n}+b_{1}^{n+1}+c_{1}^{n-1}-c_{1}^{n+2}=0
\end{gathered}
$$

for $n \in\{2,4, \ldots, 2 k-2\}$. Thus the dimension of $\operatorname{Hom}_{A^{e}}\left(\Omega^{4 k+1}(A), A\right)$ is $5 k+5$. In the same way we obtain the following sets of equations for $\operatorname{Hom}_{A^{e}}\left(\Omega^{4 k+2}(A), A\right)$ for all even $n$, such that $2 \leq n \leq 2 k$

$$
a_{1}^{n}=a_{1}^{n+1} \quad b_{1}^{n}=b_{1}^{n+1} \quad c_{1}^{n}=c_{1}^{n+1}
$$

and

$$
a_{12}=(-1)^{k} a_{21} \text { and } b_{12}=(-1)^{k} b_{21}
$$

giving $\operatorname{dim} \operatorname{Hom}_{A^{e}}\left(\Omega^{4 k+2}(A), A\right)=5 k+6$.

Finally for $\operatorname{Hom}_{A^{e}}\left(\Omega^{4 k+3}(A), A\right)$ we obtain 


$$
\begin{gathered}
a_{1}^{1}=a_{1}^{2}=\ldots=a_{1}^{2 k+2}=0 \\
b_{1}^{2}=c_{1}^{2} \\
b_{1}^{n}+b_{1}^{n+1}+c_{1}^{n-1}-c_{1}^{n+2}=0
\end{gathered}
$$

for $n \in\{2,4, \ldots, 2 k\}$ and

$$
b_{1}^{2 k+2}=(-1)^{k} a_{2} \quad \text { and } \quad c_{1}^{2 k+2}=(-1)^{k} 2 a_{2}
$$

such that $\operatorname{dim} \operatorname{Hom}_{A^{e}}\left(\Omega^{4 k+3}(A), A\right)=5 k+6$.

From Definition 2.3 we see that for $n \leq 3$ pattern for the expressions of the differential is not regular. Therefore in these small cases we have to calculate the dimension of $\operatorname{Hom}_{A^{e}}\left(\Omega^{n}(A), A\right)$ by hand. These calculations show that $\operatorname{dim} \operatorname{Hom}_{A^{e}}\left(\Omega^{1}(A), A\right)=5$, $\operatorname{dim} \operatorname{Hom}_{A^{e}}\left(\Omega^{2}(A), A\right)=6$ and $\operatorname{dim} \operatorname{Hom}_{A^{e}}\left(\Omega^{3}(A), A\right)=6$.

The dimensions of the Hochschild cohomology groups of $A$ then follow directly from above.

Theorem 3.2. The dimensions of the Hochschild cohomology groups of $A$ are as follows

$$
\operatorname{dim} \mathrm{HH}^{4 k+i}(A)= \begin{cases}2 k+3 & i=0, k>0 \\ 2 k+3 & i=1, k \geq 0 \\ 2 k+3 & i=2, k \geq 0 \\ 2 k+4 & i=3, k \geq 0\end{cases}
$$

and the set $\left\{e_{1}+e_{2}, \varepsilon, \alpha \beta+\beta \alpha, \varepsilon^{2}, \beta \alpha \beta \alpha\right\}$ is a $k$-basis of $\mathrm{HH}^{0}(A)$.

3.2. Characteristic of $k=2$. Suppose the characteristic of $k=2$. Then

Lemma 3.3. We have the following dimensions for $k>0$ :

$$
\operatorname{dim} \operatorname{Hom}_{A^{e}}\left(\Omega^{4 k+i}(A), A\right)= \begin{cases}5 k+5 & \text { if } i=0 \\ 5 k+8 & \text { if } i=1 \\ 5 k+6 & \text { if } i=2 \\ 5 k+9 & \text { if } i=3\end{cases}
$$

and $\operatorname{dim} \operatorname{Hom}_{A^{e}}\left(\Omega^{1}(A), A\right)=6, \operatorname{dim} \operatorname{Hom}_{A^{e}}\left(\Omega^{2}(A), A\right)=6$, and $\operatorname{dim} \operatorname{Hom}_{A^{e}}\left(\Omega^{3}(A), A\right)=$ 8.

Proof: The proof is analogous to the proof of Lemma 3.1 ,

Again the dimensions of the Hochschild cohomology groups of $A$ then directly follow. 
Theorem 3.4. The dimensions of the Hochschild cohomology groups of $A$ are as follows

$$
\operatorname{dim} \mathrm{HH}^{4 k+i}(A)= \begin{cases}2 k+6 & i=0, k>1 \\ 2 k+6 & i=1, k>0 \\ 2 k+6 & i=2, k>0 \\ 2 k+7 & i=3, k>0\end{cases}
$$

and $\operatorname{dim} \mathrm{HH}^{1}(A)=4, \operatorname{dim} \mathrm{HH}^{2}(A)=4, \operatorname{dim} \mathrm{HH}^{3}(A)=6$ and $\operatorname{dim} \mathrm{HH}^{4}(A)=7$. The set $\left\{e_{1}+e_{2}, \varepsilon, \alpha \beta+\beta \alpha, \varepsilon^{2}, \beta \alpha \beta \alpha\right\}$ is a k-basis of $\mathrm{HH}^{0}(A)$.

Acknowledgements. The authors would like to thank Nicole Snashall for her very helpful suggestions and comments and for the careful reading of this manuscript.

\section{REFERENCES}

[EHSST] Erdmann, K.; Holloway, M.; Snashall, N.; Solberg, Ø., Taillefer, R.' Support varieties for selfinjective algebras. K-Theory 33 (2004), no. 1, 67-87.

[EN] Erdmann, K.; Nakano, D., Representation type of Hecke algebras of type A Trans. Amer. Math. Soc. 354 (2002), no. 1, 275-285.

[ES] Erdmann, K.; Solberg, Ø., Radical cube zero weakly symmetric algebras and support varieties, preprint.

[GHMS] Green, E. L.; Hartman, G.; Marcos, E. N.; Solberg, Ø., Resolutions over Koszul algebras, Arch. Math. (Basel) 85 (2005), no. 2, 118-127.

[GS] Green, E. L.; Snashall, N., Projective bimodule resolutions of an algebra and vanishing of the second Hochschild cohomology group, Forum Math. 16 (2004), 17-36.

[GSZ] Green, E. L.; Solberg, Ø.; Zacharia, D. Minimal projective resolutions, Trans. Amer. Math. Soc. 353 (2001), no. 7, 2915-2939.

[H] Happel, D. Hochschild cohomology of finite-dimensional algebras, Springer Lecture Notes in Mathematics 1404 (1989), 108-126.

[Ho1] Holm, T. The even Hochschild cohomology ring of a block with cyclic defect group. J. Algebra 178 (1995), 317-341.

[Ho2] Holm, T. Hochschild cohomology of tame blocks. J. Algebra 271 (2004), no. 2, 798-826.

[R] Ringel, C.M. The indecomposable representations of the dihedral 2-groups. Math. Ann. 214 (1975), 19-34

[SS] Snashall, N.; Solberg, Ø., Support varieties and Hochschild cohomology rings. Proc. London Math. Soc. (3) 88 (2004), no. 3, 705-732.

[ST] Snashall, N.; Taillefer, R. The Hochschild cohomology ring of a class of special biserial algebras, preprint, arXiv:0803.1536.

Mathematical Institute, University of Oxford, 24-29 St. Giles', Oxford OX1 3LB, UK Department of MAthematics, University of Leicester, University RoAd, Leicester LE1 7RH, UK

E-mail address: erdmann@maths.ox.ac.uk, schroll@mcs.le.ac.uk 